\title{
INVESTIGACIÓN
}

Recibido: 25/07/2018 --- Aceptado: 19/06/2019 --- Publicado: 15/03/2020

\section{AUDITORÍA 2.0, UNA PERSPECTIVA PARA SU EJECUCIÓN EN EL ENTORNO EMPRESARIAL UTILIZANDO TÉCNICAS DE MINERÍA DE PROCESOS}

\author{
Audit 2.0, a perspective for its execution in the business environment \\ using process mining techniques
}

Isabel González Flores ${ }^{1}$. Universidad de las Ciencias Informáticas. Cuba. iflores@uci.cu

Josué Rivera Riquenes. Universidad de las Ciencias Informáticas. Cuba. jirivera@uci.cu

\section{RESUMEN}

La gestión empresarial necesita del desarrollo de métodos e instrumentos para establecer y mejorar las normas de actuación de las organizaciones. Un punto importante lo constituye la auditoría, que permite validar información y los procesos de negocio. En el entorno empresarial cubano la ejecución de auditorías presenta un bajo nivel de explotación de las tecnologías de la información y las comunicaciones, esto limita el trabajo de los auditores y las acciones de seguimiento y control. Las actuales prácticas se basan en muestras, lo que inevitablemente proporciona una visión incompleta de la ejecución del proceso, generalmente se realizan de forma manual, consumen mucho tiempo, así como recursos humanos y financieros. El objetivo de este trabajo es destacar los beneficios del análisis de la información disponible en los registros de eventos de los sistemas informáticos, utilizando técnicas de Minería de procesos como una nueva forma de auditoría. La automatización de la auditoría reduce los costos de transacción correspondientes, mejorando al mismo tiempo su calidad y precisión, así lo demuestran los casos de estudio analizados. Las organizaciones que asuman esta nueva forma de auditoría para sus procesos de negocio tendrán asegurado un mayor nivel de competitividad frente a sus adversarios en el mercado actual.

PALABRAS CLAVE: auditoría - minería de procesos - registro de eventos sistemas de información - organizaciones - procesos de negocio - tecnologías de la información.

\footnotetext{
${ }^{1}$ Isabel González Flores: Máster en Tecnologías de Apoyo a la Toma de Decisiones Empresariales de la Facultad de Ingeniería Industrial de la CUJAE en 2015. Graduada de la Universidad de las Ciencias Informáticas (UCI) en 2010. Posee 6 años de experiencia como analista de software.
} 


\section{ABSTRACT}

Business management needs the development of methods and instruments to establish and improve the performance standards of organizations. An important point is the audit, which allows validating information and business processes. In the Cuban business environment, the execution of audits shows a low level of exploitation of information and communication technologies, which limits the work of the auditors and the follow-up and control actions. The current practices are based on samples, which inevitably provide an incomplete view of the execution of the process, which are usually done manually; consume a lot of time, as well as human and financial resources. The objective of this work is to highlight the benefits of the analysis of the information available in the registers of events of the computer systems, using techniques of Process Mining as a new form of audit. The automation of the audit reduces the corresponding transaction costs, while improving its quality and accuracy, as the case studies analyzed show.

KEY WORDS: audit - process mining - event log - information systems organizations - business processes - information technology.

\section{AUDITORIA NÚMERO 2.0, UMA PERSPECTIVA PARA SUA EXECUÇÃO EM UM ENTORNO EMPRESARIAL UTILIZANDO TÉCNICAS DE EXPLORAÇÃO DE DADOS}

\section{RESUME}

A gestão empresarial necessita do desenvolvimento de métodos e instrumentos para estabelecer e melhorar as normas de atuação das organizações. Um ponto importante constitui a auditoria, que permite validar informação e os processos de negócio. No entorno empresarial cubano a execução de auditorias apresenta um baixo nível de exploração das tecnologias da informação e as comunicações, isso limita o trabalho dos auditores e as ações de seguimento e controle. As atuais praticas se baseiam em amostras, o que inevitavelmente proporciona uma visão incompleta da execução do processo, geralmente se realizam de forma manual, consomem muito tempo, assim como recursos humanos e financeiros. O objetivo deste trabalho é destacar os benefícios das análises da informação disponível nos registros de eventos dos sistemas informativos, utilizando técnicas de Extração de processos como uma nova forma de auditoria. A automatização da auditoria reduze os custos de transação correspondentes, melhorando ao mesmo tempo sua qualidade e precisão, assim demonstram os casos de estudo analisados. As organizações que assumam esta nova forma de auditoria para seus processos de negócio terão assegurado um maior nível de competitividade diante de seus adversários no mercado atual.

PALAVRAS CHAVE: auditoria - extração de processos - registros de eventos sistemas de informação - organizações - processos de negócio - tecnologias da informação. 


\section{Como citar el artículo:}

González Flores, I. y Rivera Riquenes, J. (2020). Auditoría 2.0, una perspectiva para su ejecución en el entorno empresarial utilizando técnicas de minería de procesos. [Audit 2.0, a perspective for its execution in the business environment using process mining techniques]. Vivat Academia. Revista de Comunicación, 150, 47-57.

doi: http://doi.org/10.15178/va.2020.150.47-57

Recuperado de http://www.vivatacademia.net/index.php/vivat/article/view/1129

\section{INTRODUCCIÓN}

El contexto actual, caracterizado por la competitividad empresarial, la globalización y acelerados cambios científicos y tecnológicos, la gestión empresarial necesita del desarrollo de métodos e instrumentos para establecer y mejorar las normas de actuación de las organizaciones. En (Yzquierdo Herrera, 2013) se refleja que una de las principales prioridades de las organizaciones se relaciona con la mejora de sus procesos apoyados en el uso de las tecnologías de la información y las comunicaciones, por lo general tienen una tendencia a enfocarse en la implementación y modelado de procesos, pero en muchas ocasiones ocurre que tienen poco conocimiento sobre sus propios procesos.

El desarrollo de la auditoría incide de forma directa en la eficacia del sistema de gestión de la organización y su mejora. Su integración en diferentes dimensiones (documentación, procesos y recursos humanos) confluye en un Sistema Integrado de Gestión (Karapetrovi et al., 2010). En la literatura aparecen un grupo de investigaciones asociadas al desarrollo de procedimientos, metodologías o guías de auditoría con objetivos variados, entre ellos, auditoría logística, de recursos humanos, de información, entre otros; en algunos casos asociadas a modelos teóricos, entre las que se subrayan los propuestos por (Delgado Pérez, 2002), (Sotolongo Sánchez, 2005), (Goñi Camejo, 2008) y (Acosta Palmer y Troncoso Fleitas, 2011).

En las propuestas mencionadas se limita, desde una perspectiva metodológica, la disponibilidad de herramientas que orienten a los auditores en la aplicación del enfoque de calidad, sobre la cual no se perciben evidencias asociadas a indicadores de eficacia que expresen de forma cuantitativa su estado en función de asegurar el cumplimiento de los objetivos. A pesar del reconocimiento de los aportes de las investigaciones anteriores, en la gestión de auditoría, existen insuficiencias relacionadas con el bajo nivel de explotación de las tecnologías de la información y las comunicaciones, expresado en la ausencia de sistemas de información que implementen los modelos y procedimientos existentes e integren herramientas para la ejecución de este proceso con la calidad requerida (Escobar Rivera et al., 2016).

Los auditores validan la información sobre las organizaciones y sus procesos de negocio. Tradicionalmente, una auditoría sólo puede proporcionar una garantía razonable de que los procesos de negocio se ejecutan dentro de los límites establecidos (Van der Aalst et al., 2010). Grandes escándalos corporativos y de 
González Flores, I. y Rivera Riquenes, J.

Auditoría 2.0, una perspectiva para su ejecución en el entorno empresarial utilizando técnicas de minería de procesos

contabilidad incluidos los que afectan Enron, Tyco, Adelphia, Peregrine y WorldCom han impulsado el interés en las prácticas de auditorías más rigurosas (Van der Aalst, 2016).

En el entorno empresarial cubano la ejecución de auditorías presenta un bajo nivel de explotación de las tecnologías de la información y las comunicaciones, esto limita el trabajo de los auditores y las acciones de seguimiento y control. Las actuales prácticas se basan en entrevistas con las personas de la organización y en muestras, lo que inevitablemente proporciona una visión incompleta de la ejecución del proceso. Generalmente se realizan de forma manual, consumen mucho tiempo, así como recursos humanos y financieros. Estas deficiencias no solo se presentan en el entorno cubano, también son mencionadas en (Sayana SA, 2003), (Gallegos y Carlin, 2007), (Stocker T y Müller, 2013), (Werner, 2016). Además, las auditorías a procesos informatizados deberían ser auditadas por herramientas de igual tipo y no manualmente.

\section{OBJETIVOS}

El objetivo de este trabajo es destacar los beneficios del análisis de la información disponible en los registros de eventos de los sistemas informáticos, utilizando técnicas de Minería de procesos como una nueva forma de auditoría.

\section{METODOLOGÍA}

Constantemente se registra información sobre los procesos de negocios, por los sistemas de información, pero es una información que por lo general se usa poco (Yzquierdo Herrera, 2013). La ciencia de los datos es la profesión del futuro, porque las organizaciones que no pueden usar datos de una manera inteligente no sobrevivirán. Los registros de eventos se encuentran disponibles en los sistemas de información en los que se identifican los procesos del negocio, conocidos en la literatura por el acrónimo en inglés PAIS (Process Aware Information Systems), como son los sistemas de workflow, BPMS, ERP - Enterprise Resource Planning, CRM, entre otros.

La Minería de procesos es una disciplina que tiene como objetivo descubrir, monitorear y mejorar procesos a través de la extracción de conocimiento del registro de eventos de los sistemas de información (Van der Aalst, Process Mining. Discovery, Conformance and Enhancement of Business Processes, 2011) (Yzquierdo Herrera, 2013). Puede aplicarse a cualquier tipo de procesos operativos, sus positivas contribuciones para aplicarse en la ejecución de auditorías son discutidas en (Jans et al., 2014), (Van der Aalst et al., 2010), (Werner y Gehrke, 2015), (Jans, Alles,, \& MA, 2014), (Werner, 2016).

La modelación tradicional de los procesos de negocio que son implementados por sistemas informáticos representa lo que se debe hacer y está sujeta a errores por la presencia del factor humano. A diferencia, la Minería de procesos muestra lo que 
González Flores, I. y Rivera Riquenes, J.

Auditoría 2.0, una perspectiva para su ejecución en el entorno empresarial utilizando técnicas de minería de procesos

realmente sucede, por lo que se puede hacer el análisis del funcionamiento de los procesos de una organización, basado en los hechos que ya ocurrieron y que se encuentran guardados en los registros de eventos. En la figura 1 se presentan los tipos de Minería de procesos (Van der Aalst et al., 2010),

- Descubrimiento: Las técnicas de Minería de procesos pueden extraer, a partir de la información contenida en los registros de eventos, los patrones más frecuentes y reflejarlos en los modelos que describen los procesos manejados (Van der Aalst y Van Dongen, 2002) (de Medeiros et al., 2004) (Van der Aalst y Weijters T, 2004). Con el modelo generado, al que llamaremos modelo teórico, el auditor puede tener una visión imparcial de lo que realmente ha sucedido. Entre los principales algoritmos para el descubrimiento se encuentran: Fuzzy Miner (Günther y Van der Aalst, 2007), Alpha Miner (Van der Aalst, 2011), Heuristic Miner (Weijters y Ribeiro, 2011) y Genetic Miner (de Medeiros et al., 2007).

- Verificación de conformidad: Un auditor puede utilizar el modelo teórico del proceso para comprobar si la realidad (según la información en el registro de eventos) está conforme al modelo y viceversa, lo que resulta de gran utilidad para detectar desviaciones, localizarlas, explicarlas y medir su gravedad.

- Mejoramiento: También conocido como extensión, permite extender el modelo teórico de procesos de la empresa, a partir de la consideración de la información contenida en el registro de eventos para enriquecer el modelo.

Principales herramientas para la Minería de procesos:

- ProM: al ser una herramienta de código abierto y de libre distribución ha sido utilizada para la Minería de procesos. Permite el proceso de descubrimiento, la comprobación de la conformidad, análisis de redes sociales, la minería de organización, la minería de decisión (Van der Aalst, 2011), (Process Mining Group, 2010). No está enfocada en la usabilidad, por lo que requiere de usuarios con experiencia en la Minería de procesos.

- Disco: se considera es la herramienta privativa más utilizada para la Minería de procesos. Desarrollada por Fluxicon en el 2009 (Laboratories Fluxicon Process, 2017), la licencia gratuita con fines académicos presenta limitaciones para su uso. Es compatible con las versiones 5.0 y 6.0 de ProM.

Las restantes herramientas estudiadas son de carácter comercial: QPR Process Analyzer (QPR Software Oyj, 2017), ARIS Process Performance Manager (Fischer, 2008), ver otros en (Van der Aalst et al., 2011).

\section{RESULTADOS}

A continuación se presentan casos de estudio en los que se ha utilizado la Minería de procesos para la ejecución de auditorías.

- En (Yzquierdo Herrera, 2013) se presenta un caso de estudio desarrollado en Cuba para el Sistema Único de Identificación Nacional (carné de identidad). Caso de estudio modelado del proceso de gestionar los roles de la solución, 
contiene 31 casos, 804 eventos. Se obtuvo un modelo representativo del proceso aplicando el algoritmo Alpha++. Se emplea la técnica denominada Advanced Dotted Chart Analysis, implementada como parte de la herramienta ProM, para realizar un diagnóstico visual del proceso analizado. Como resultado se determinó que la tarea que fallaba con mayor frecuencia era Condicionar Operación, la cual se ejecutó en 31 ocasiones y falló el 38,7\% de las veces. Se identificaron 60 eventos de tipo fallo, por lo cual los fallos asociados a la tarea Condicionar Operación representan el $20 \%$ de los fallos ocurridos.

- En (Jans et al., 2014) se presenta un caso de estudio del sector bancario en el que se utilizaron 26185 instancias del proceso, relacionado con los pagos dispuestos por 272 trabajadores de un banco europeo. Se aplica el algoritmo de descubrimiento Fuzzy Miner y técnicas de análisis de redes sociales, que permitieron identificar pagos realizados sin previa autorización y violaciones de procedimientos internos de la compañía. Estas anomalías no fueron detectadas por los procedimientos convencionales de auditoría realizados por auditores internos sobre los mismos datos.

- En (Jans et al., 2012) se tuvo acceso a datos que habían sido auditados por los auditores internos de una empresa, proporcionando así un punto de referencia para evaluar la contribución incremental de la Minería de procesos al destapar información relevante de auditoría no detectada previamente por la norma Procedimientos de auditoría. Después de la aplicación de la Minería de procesos se descubren fallas de control interno que los propios auditores internos de la empresa no pudieron detectar. Se identificaron transacciones relevantes de auditoría que justificaban una investigación más profunda por parte de los auditores internos: tres órdenes de compra que pasaron por el proceso de adquisición sin ninguna señal o liberación, en violación de los procedimientos de control, 175 violaciones del principio de separación de funciones que exige que las entradas y salidas de mercancías, 265 pagos que no tienen la factura correspondiente.

- En (De Weerdt et al., 2013) se presenta un caso de estudio del sector financiero, específicamente referente a una compañía de seguros Belga. Para el análisis de control de flujo de los documentos de seguros que se gestionan se realizó el análisis de 34.769 casos que contemplan 15 actividades empresariales reales, correspondientes a un período de seis meses. Con el algoritmo Heuristics Miner visualizó el proceso, que indica una fuerte libertad de comportamiento que permite el sistema de información existente. Se identificaron errores de clasificación de documentos e ineficiencias en el frecuente traspaso de documentos.

- En (Van der Aalst et al., 2007) se describe la aplicación de la minería proceso en una de las oficinas provinciales del Departamento de Obras Públicas Nacional de Holanda, responsable de la construcción y el mantenimiento de la infraestructura vial y de agua. El análisis la tramitación de las facturas enviadas por los diversos subcontratistas y proveedores, con auxilio de la Minería de procesos, permitió identificar ciclos de trabajos no deseados que 
tienen un gran impacto en el rendimiento del proceso. Al respecto, se identificaron los roles y malas prácticas que afectan fuertemente el desempeño del proceso en términos de tiempo.

- En (Ramírez Pérez, 2016) se presenta un modelo para la selección de equipos de trabajo quirúrgico en sistemas de información en salud aplicando técnicas de inteligencia organizacional y la Minería de procesos. Los estudios realizados demuestran que en los hospitales no era posible la selección efectiva de los equipos de trabajo quirúrgico, debido a que la información obtenida en el proceso de atención al paciente no se gestiona adecuadamente, no se analiza la interacción entre las personas a partir de los datos existentes y no se potencian análisis para mejorar la selección de los equipos de trabajo y la calidad de las intervenciones quirúrgicas. Los resultados del proyecto de investigación fueron llevados a cabo en el Hospital Dr. Gustavo Aldereguía Lima de Cienfuegos y, posteriormente, se validó en ocho hospitales de cuatro provincias del país, seleccionados a partir de su representatividad en el Sistema Nacional de Salud. La aplicación de técnicas de inteligencia organizacional y la Minería de procesos permitieron evidenciar una disminución en la ocurrencia de intervenciones quirúrgicas no satisfactorias y la disminución, hasta tres horas, del tiempo empleado para la realización de tales procedimientos. Un análisis económico del ahorro que implicaría la aplicación del modelo muestra que solamente para una operación con procedimiento quirúrgico: ictus isquémico agudo, se reduciría el costo de $\$ 9674$ a $\$ 5608$.

Desde el 2010 en (Van der Aalst et al., 2010) se plantea que con la información detallada de los procesos, cada vez más disponibles en los registros de eventos de alta calidad, los auditores ya no tienen que depender de un pequeño conjunto de muestras. En su lugar, utilizando técnicas de Minería de procesos, pueden evaluar todos los eventos en un proceso empresarial y hacerlo mientras aún se están ejecutando. La omnipresencia de los eventos de negocios registrados electrónicamente junto con la tecnología de Minería de procesos permite una nueva forma de auditoría que cambiará drásticamente el rol de los auditores: Auditoría 2.0.

\section{DISCUSIÓN}

A partir de los casos de estudio presentados se evidencia que la Minería de procesos es una disciplina novedosa que puede ser aplicada para el desarrollo de auditorías. La detección de comportamientos irregulares en los procesos de negocio permite a los directivos adoptar las medidas organizativas para realizar mejoras en los procesos. En (Yzquierdo Herrera, 2013) se afirma que la Minería de procesos permite realizar un estudio analítico basado en información pertinente, en hechos, pues con la abundancia de información, hoy esto no es una utopía, es una realidad. Según la experticia de los autores de los casos de estudios mencionados, para que el análisis sea pertinente:

- Las actividades que se realizan fuera de los sistemas de información no pueden ser analizadas. 
González Flores, I. y Rivera Riquenes, J.

Auditoría 2.0, una perspectiva para su ejecución en el entorno empresarial utilizando técnicas de minería de procesos

- La calidad de los datos extraídos es directamente proporcional al resultado del análisis, debiéndose garantizar su confiabilidad, completitud y validez.

- Los sistemas de información no registran los datos de eventos ni usan las mismas estructuras de almacenamiento de datos de forma homogénea, por tanto para la extracción del registro de eventos es crucial el trabajo conjunto del analista de Minería de procesos y los especialistas pertinentes de la organización.

- En el análisis de los resultados deben participar los trabajadores de la organización que dominan el negocio, en aras de tener una visión acertada de cada contexto.

- La selección de las técnicas y algoritmos para el análisis con Minería de procesos debe estar asociada a los objetivos del proyecto y a las preguntas que se quieran resolver (Aguirre Mayorga y Rincón García, 2015).

Las técnicas de Minería de procesos ofrecen un medio para una comprobación del cumplimiento más riguroso y determinar la validez y fiabilidad de la información acerca de los procesos fundamentales de una organización (Van der Aalst, 2016). Sus potencialidades han sido modestamente empleadas en el entorno cubano para la ejecución de auditorías. Esto se puede deber a que no existe suficiente cultura en su uso ni en el desarrollo de sistemas de información que identifiquen los procesos del negocio. Además, es importante mencionar que las principales referencias bibliográficas sobre este tema se encuentran en idioma inglés.

\section{REFERENCIAS}

Acosta Palmer, H. y Troncoso Fleitas, M. (2011). Auditoría integral de mantenimiento en instalaciones hospitalarias, un análisis objetivo. Ingeniería Mecánica, 14(2), 107 118.

Aguirre Mayorga, H. y Rincón García, N. (2015). Minería de procesos: desarrollo, aplicaciones y factores críticos. Cuadernos de Administración, 28(50), 137-157.

de Medeiros, A., Weijters, A. J. \& Van der Aalst, W. (2007). Genetic process mining: an experimental evaluation. Data Mining and Knowledge Discovery, 14(2), 245-304. doi: https:// doi.org/10.1007/s10618-006-0061-7

de Medeiros, A., Dongen, V. B., Van der Aalst, W. \& Weijters, A. (2004). Process mining: extending the alpha-algorithm to mine short loops. Technische Universiteit Eindhoven.

De Weerdt, J., Schupp, A., Vanderloock, A. \& Baesens, B. (2013). Process Mining for the multi-faceted analysis of business processes - A case study in a financial services organization. Computers in Industry, 64(1), 57-67. 
González Flores, I. y Rivera Riquenes, J.

Auditoría 2.0, una perspectiva para su ejecución en el entorno empresarial utilizando técnicas de minería de procesos

Delgado Pérez, E. (2002). Metodología para la realización del diagnóstico de la Gestión de los Recursos Humanos en empresas en Perfeccionamiento Empresarial. (Tesis inédita de maestría). Universidad de Holguín: Oscar Lucero Moya, Holguín, Cuba.

Escobar Rivera, D., Moreno Pino, M. y Cuevas Rodríguez, L. (2016). La calidad de la auditoría en Sistemas de Gestión. Software AUDIT_INTEGRATED. Ciencias Holguín, 22(2), 77-93.

Fischer, M. (2008). ARIS Process Performance Manager. 14th GI/ITG ConferenceMeasurement, Modelling and Evalutation of Computer and Communication Systems, 1-3.

Gallegos, F. \& Carlin, A. (2007). IT Audit: A Critical Business Process. Computer, $40(7), 87-9$.

Goñi Camejo, I. (2008). El qué y el cómo del diagnóstico del sistema de información gerencial. ACIMED, 17(5), 1-19.

Günther, C. \& Van der Aalst, W. (2007). Fuzzy Mining - Adaptive Process Simplification Based on Multi-perspective Metrics. Business Process Management, 328-343. Recuperado de: https://link.springer.com/chapter/10.1007/978-3-54075183-0_24

Jans, A. M. \& MA, V. (2014). A Field Study on the Use of Process Mining of Event Logs as an Analytical Procedure in Auditing. Accounting Review, 89(5), 1751-1773.

Jans, M., Alles, M. \& Vasarhelyi, M. (2012). Process mining of event logs in internal auditing: a case study. Recuperado de https://uhdspace.uhasselt.be/dspace/handle/1942/14227

Jans, M., Alles, M. \& Vasarhelyi, M. (2014). A Field Study on the Use of Process Mining of Event Logs as an Analytical Procedure in Auditing. Accounting Review, 89(5), 1751-1773.

Karapetrovi, S., Casadesus, M. \& Heras, I. (2010). Empirical analysis of integration within the standards-based integrated management systems. Int J Qual Res, 4(1), 25-35.

Laboratories Fluxicon Process. (2017). Process Mining and Automated Process Discovery Software for Professionals - Fluxicon Disco. Recuperado de: https://fluxicon.com/disco/

Process Mining Group, E. T. (2010). ProM 6 tutorial. Recuperado de: http://www.promtools.org/doku.php?id=tutorial:start 
González Flores, I. y Rivera Riquenes, J.

Auditoría 2.0, una perspectiva para su ejecución en el entorno empresarial utilizando técnicas de minería de procesos

QPR Software Oyj. (2017). QPR ProcessAnalyzer. Recuperado de: https://www.qpr.com/node/4

Ramírez Pérez, J. (2016). Modelo para la selección de equipos de trabajo quirúrgico en sistemas de información en salud aplicando técnicas de inteligencia organizacional. (Tesis inédita de doctorado). Universidad de las Ciencias Informáticas, La Habana, Cuba.

Sayana SA. (2003). Using CAATs to Support IS Audit. Inf Syst Control J. Recuperado de

http:/ / csbweb01.uncw.edu/people/IvancevichD/classes/MSA\%20516/Extra\%20 Readings\%20on\%20Topics/CAATS/Supplemental\%20Reading\%20Week\%208/U sing $\%$ 20CAATTS $\% 20$ to $\% 20$ Support $\% 20 I T \% 20$ Audit.pdf

Sotolongo Sánchez, M. (2005). Procedimiento para la auditoria interna del Sistema de Gestión de Recursos Humanos en instalaciones turísticas hoteleras cubanas. Aplicación en pequeñas y medianas instalaciones turísticas hoteleras. (Tesis inédita de doctorado). Universidad Marta Abreu de Las Villas, Santa Clara, Villa Clara, Cuba.

Stocker T, R. \& Müller, G. (2013). On the Exploitation of Process Mining for Security Audits: The Process Discovery Case. Proceedings of the 28th Annual ACM Symposium on Applied Computing, (pp. 1462-1468). Recuperado de http:// doi.acm.org/10.1145/2480362.2480634

Van der Aalst, W. (2011). Do Petri Nets Provide the Right Representational Bias for Process Mining? ART@ Petri Nets, (pp. 85-94). Newcastle upon Tyne, UK: J. Desel \& A. Yakovlev.

Van der Aalst, W. (2011). Process Mining. Discovery, Conformance and Enhancement of Business Processes, (pp. 85-94). Springer-Verlag Berlin Heidelberg.

Van der Aalst, W. (2016). Process Mining: Data Science in Action, (477). Springer.

Van der Aalst, W. \& Van Dongen, B. (2002). Discovering Workflow Performance Models from Timed Logs. Van der Aalst, W., \& Van Dongen, B. (2002). Discovering Workflow Performance Models from Timed Logs. Engineering and Deployment of Cooperative Information Systems, (pp. 45-63). Recuperado de http://link.springer.com/chapter/10.1007/3-540-45785-2_4

Van der Aalst, W. \& Weijters T, M. L. (2004). Workflow mining: discovering process models from event logs. IEEE Trans Knowl Data Eng, 16(9), 1128-1142.

Van der Aalst, W., Adriansyah, A., de Medeiros, A., Arcieri, F., Baier, T. \& Blickle, T. (2011). Process Mining Manifesto. Business Process Management Workshops, 169- 
González Flores, I. y Rivera Riquenes, J.

Auditoría 2.0, una perspectiva para su ejecución en el entorno empresarial utilizando técnicas de minería de procesos

194. Recuperado de https://link.springer.com/chapter/10.1007/978-3-642-281082_19

Van der Aalst, W., Reijers, H., Weijters, A., Van Dongen, B., de Medeiros, A. S., et al. (2007). Business process mining: An industrial application. Information Systems, 32(5), 713-732. doi: http://doi.acm.org/10.1016/j.is.2006.05.003

Van der Aalst, W., van Hee, K., van Werf, J. \& Verdonk, M. (2010). Auditing 2.0: Using Process Mining to Support Tomorrow's Auditor. Computer, 43(3), 90-93.

Weijters, A. \& Ribeiro, J. (2011). Flexible Heuristics Miner (FHM). IEEE Symposium on Computational Intelligence and Data Mining (CIDM), 310.

Werner, M. (2016). Process Model Representation Layers for Financial Audits. 49th Hawaii International Conference on System Sciences (HICSS), (pp. 5338-5347). USA.

Werner, M. \& Gehrke, N. (2015). Multilevel Process Mining for Financial Audits. IEEE Trans Serv Comput, 8(6), 820-832.

Yzquierdo Herrera, R. (2013). Minería de proceso como herramienta para la auditoria. Ciencias de la Información, 44(2), 25-32.

\section{AUTORES:}

\section{Isabel González Flores}

Graduada de la Universidad de las Ciencias Informáticas (UCI) en 2010, máster en Tecnologías de Apoyo a la Toma de Decisiones Empresariales de la Facultad de Ingeniería Industrial de la CUJAE en 2015. Profesor instructor de la asignatura Metodología de la Investigación Científica. Experiencia de seis años como analista de software del Proyecto de Informatización para la Gestión de los Tribunales Populares Cubanos. Miembro del grupo de investigación de Minería de procesos de la UCI. Líneas de investigación: Informática jurídica, Minería de procesos, Tecnologías de Apoyo a la Toma de Decisiones Empresariales. Ha tutorado seis trabajos de diploma de pregrado.

ResearchGate: https://www.researchgate.net/profile/Isabel_Gonzalez_Flores

\section{Josué Rivera Riquenes}

Graduado de la Universidad de las Ciencias Informáticas (UCI) en 2013, profesor instructor de la asignatura Gráficos por Computadoras. Certificado Microsoft Technology Associate (MTA) for Database Administration Fundamentals. Experiencia de 5 años como desarrollador de software del Proyecto de Informatización para la Gestión de los Tribunales Populares Cubanos. Miembro del grupo de investigación Informática Jurídica y del grupo de investigación de Minería de procesos de la UCI. Las principales líneas de investigación relacionadas con el uso de las TIC son: Informática jurídica, Minería de procesos y desarrollo con tecnologías Arduino. Ha sido tutor de un trabajo de diploma de pregrado.

ResearchGate: https://www.researchgate.net/profile/Josue_Rivera_Riquenes 